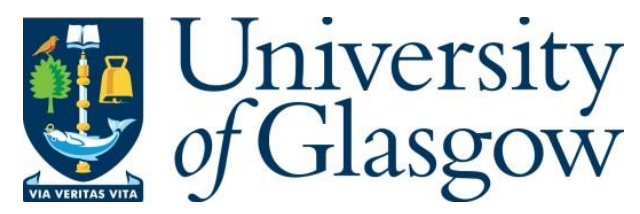

O'Hagan, J., Williamson, J. R. and Khamis, M. (2020) Bystander Interruption of VR Users. In: 9th ACM International Symposium on Pervasive Displays, New York, NY, USA, 4-5 Jun 2020, pp. 19-27. ISBN 9781450379861

(doi:10.1145/3393712.3395339).

There may be differences between this version and the published version. You are advised to consult the publisher's version if you wish to cite from it.

(C) The Authors 2020. This is the author's version of the work. It is posted here for your personal use. Not for redistribution. The definitive Version of Record was published in Proceedings of the 2020 ACM Conference on Innovation and Technology in Computer Science Education (ITiCSE '20), Trondheim, Norway, 1519 Jun 2020, p. 567. ISBN 9781450368742.

http://eprints.gla.ac.uk/217034/

Deposited on: 29 May 2020

Enlighten - Research publications by members of the University of Glasgow http://eprints.gla.ac.uk 


\section{Bystander Interruption of VR Users}

\author{
Joseph O'Hagan \\ University of Glasgow \\ Glasgow, United Kingdom \\ j.ohagan.1@ research.gla.ac.uk
}

\author{
Julie R. Williamson \\ University of Glasgow \\ Glasgow, United Kingdom \\ julie.williamson@glasgow.ac.uk
}

\author{
Mohamed Khamis \\ University of Glasgow \\ Glasgow, United Kingdom \\ mohamed.khamis@glasgow.ac.uk
}

\begin{abstract}
Research has begun to investigate the interruption of VR users but lacks an understanding of how social factors (setting / relationship to the VR user) might impact the interruption and why bystanders interrupt as they do. We conducted a survey $(\mathrm{N}=100)$ into bystander comfort when interrupting a VR user (known / unknown) in 4 settings (private spaces, public spaces, private transport, public transport) and their willingness to use a range of interruption strategies. Our results suggest relationship to the VR user is more influential than setting when considering comfort and acceptability of interruption strategy. A follow-up lab study $(\mathrm{N}=16)$ investigated bystander interruption of a known VR user in a private setting. Most used a combination of speech and touch to interrupt though a subset used unconventional, improvised strategies we term playful interruptions. With the recent inclusion of open ear audio in VR headsets and preference towards verbal interruptions in our results we conducted a survey $(\mathrm{N}=76)$ into the consumer response to open ear audio in VR headsets. We report open ear audio was used regularly by $52.6 \%$ of our respondents $60 \%$ of who rate their experience with it positively.
\end{abstract}

\section{Author Keywords}

Head-Mounted Displays; Virtual Reality; Interruptions

\section{INTRODUCTION}

With the rise of virtual reality (VR) headset usage, interactions between VR users and bystanders are becoming more frequent $[8,9,12,14,24]$. Recent research has investigated how the interruption of VR users can be eased by increasing a VR user's awareness of nearby people [5, 7, 13, 25] while others have studied the interruption process itself $[3,4]$. At present it is not known how factors like the interrupter's relationship to the VR user or setting can impact the interruption. To this end, we present a survey $(\mathrm{N}=100)$ which investigated bystander comfort when interrupting a VR user (known / unknown) across 4 settings (private spaces, public spaces, private transport, public transport) and their willingness to use potential interruption strategies. Our results indicate a bystander's relationship to the VR user is more influential than setting when considering comfort and choice of interruption strategy.

This is the author's version of the work. It is posted here for your personal use. Not for redistribution. The definitive Version of Record was published in the 9th ACM International Symposium on Pervasive Displays (PerDis '20), June 4-5.

https: //doi .org/10.1145/3393712.3395339
Follow-up work investigated what motivates bystanders to interrupt as they do. We conducted a lab study $(\mathrm{N}=16)$ into bystander interruption for known VR users in a private setting. We found the combination of speech and touch was the preferred interruption approach. Participants said they did not want to surprise or scare the VR user when interrupting and believed verbally announcing their presence and touching to provide a reference of their location would minimise the VR user's discomfort. We also observed the emergence of improvised strategies, we term playful interruptions, where participants improvised an unconventional, novel approach.

Motivated by the use of verbal interruptions in our results and the adoption of open ear audio, where audio is provided but the user's ears are left uncovered, in VR headsets [17, 18, 19, 32] we conducted a survey $(\mathrm{N}=76)$ into the consumer response to open ear audio in VR headsets. Prior work found most VR users to use headphones exclusively [13] and we were curious if open ear audio had altered this. We report open ear audio was used regularly by $52.6 \%$ of our respondents $60 \%$ of who rate their experience with it positively.

\section{RELATED WORK}

Research on how to ease the interruption of VR users can be categorised into two approaches:

- Bidirectional: Attempts to establish a bidirectional interaction between the VR user and bystander. Provides the VR user with sufficient awareness of their surroundings to allow them to initiate an interaction with a bystander.

- Unidirectional: Attempts to maintain a VR user's sense of presence until the moment of interruption. Leaves the onus on the bystander to initiate an interaction with a VR user.

\section{Bidirectional Interruptions}

Attempts to increase a VR user's awareness of reality have envisioned how a VR headset might be capable of identifying, interpreting and responding to a user's surrounding area. McGill et al. proposed increasing awareness of copresence by automatically detecting and including photorealistic silhouettes of bystanders within the virtual environment [13]. Their approach was found to significantly disrupt the VR user's sense of presence and they concluded less distracting notifications were needed. Building on this work, Willich et al. compared 3 visualisations for the bystander: an abstract avatar, a photorealistic silhouette and a passthrough video of the bystander and some of the surrounding environment [4]. They found users preferred the aesthetic of the avatar or silhouette over the passthrough video and reported the avatar 
was the most accurate visualisation to allow a VR user to locate the bystander's position relative to their own. Simeone developed a motion tracking widget to plot the position of bystanders relative to the VR user but conducted a limited pilot test evaluation of his work [25]. Ghosh et al. investigated which elements of reality VR users wanted to be informed about. They identified 13 scenarios, 8 of which concerned a desire for increased auditory awareness and 5 for increased awareness of the surrounding physical space [7]. Ghost et al. also conducted a lab study comparing notifications from 6 modalities (audio, visual, haptic, audio + haptic, visual + haptic, audio + visual) for 5 usage scenarios (person talking, incoming call, incoming message, person in room, time spent in VR) in terms of noticeability and perception but did not investigate what impact they had on a user's sense of presence.

Consumer VR headsets have taken a different approach and adopted open ear audio designs where the on-board system audio leaves the user's ears uncovered to increase their auditory awareness of the surrounding area. The first implementation of open ear audio in a VR headset was the Oculus Go [17] which integrated speakers into the headset's headband, a design reused by the Rift S [19] and Quest [18] headsets. The Valve Index [32] meanwhile positioned open back headphones next to, but not touching, the user's ears. The response to open ear audio VR headsets remains unclear. Critics praise it for increasing a user's awareness but say headphones are necessary for immersive audio [6] while online comments range from "the new audio system is much more immersive" [29] to “WORST AUDIO DECISION EVER” [11].

\section{Unidirectional Interruptions}

Unidirectional interruption systems, which require the bystander interrupt the VR user, are already present in VR headsets. The first was the HTC Vive "Knock" notification [31], a text notification to pause the application in use and notify the VR user that someone nearby desires their attention. Triggering the notification requires the bystander to use the PC the HTC Vive is connected to though one user has customised their system to allow remote triggering via a bluetooth remote [28]. Zenner et al. extended this idea and proposed allowing bystanders to send text notifications to the VR user [34] with messages being sent as a low, medium and high priority which were represented differently in VR. Zenner et al. did not evaluate their work but research on perceptual phenomenon, change blindness and Rzayev et al's work on VR notification design $[22,26]$ suggest the low priority notifications would go unnoticed while the medium / high priority notifications would be noticed but significantly disrupt the user's sense of presence [3, 13, 23, 27]. Williamson et al. investigated methods a passenger using a VR headset on an airplane might be interrupted [33]. They proposed the use of a passthrough view and a peripheral device to allow bystanders to trigger an interruption and concluded a range of interruption techniques are necessary to accommodate a range of potential usage scenarios.

\section{Investigating the Interruption Process}

George et al. have investigated the interruption process itself. They first considered the interplay of presence, workload and attention when designing interruption notifications [3]. They compared 3 notifications across 2 settings and discussed the trade-off between presence and attention when designing VR interruptions. Their results found notifications designed to be more attention grabbing negatively impacted sense of presence in VR. Follow-up work showed bystanders can identify when a VR user switches task within VR which they argued was the optimal time for an interruption to occur [4]. George et al. reported participants preferred to use speech or touch when interrupting but did not explore why participants used their respective strategies or their comfort while interrupting.

\section{SURVEY: INTERRUPTIONS IN THEORY}

We developed a survey to investigate the comfort of bystanders when interrupting a VR user and their willingness to use 5 potential interruption strategies. Our survey had 4 settings motivated by prior work: private spaces, public spaces, private transport, public transport $[8,9,12,14,24,33]$. For each setting respondents were given a picture (Appendix 1) and text description. Respondents were asked to imagine interrupting a VR user who was their friend in the given setting and asked to rate their comfort when interrupting using a 5-point scale ( $1=$ very uncomfortable; $5=$ very comfortable). Respondents were then asked to indicate their willingness (Yes / No) to use each of the following when interrupting: 1) Make physical contact with them, 2) Speak to them, 3) Wave or gesture at them, 4) Use a purpose built peripheral for attracting their attention, and 5) Press a key on a nearby keyboard. An open text field "other" was included to allow respondents to propose their own approach. Speech and touch were included as conventional social interruption techniques bystanders are familiar with. Gestures were included as an alternative social interruption to allow interruptions from afar. A purpose built peripheral was positively reported by Williamson et al. [33]. The option to use a keyboard was included as an example of a peripheral bystanders would be familiar with. Respondents were then asked to imagine the VR user being interrupted was a stranger and the questions repeated.

\section{Limitations}

Survey respondents may have misinterpret the proposed interruption strategy "wave or gesture at them" as a trick question as VR users would not be able to see their gestures. While we attempted to convey their wave or gesture would trigger an interruption notification respondents may have misunderstood.

\section{SURVEY RESULTS}

We distributed the survey through mailing lists and social media. 100 (40 female) respondents aged between 16 and 60 $(\mathrm{M}=27.45, \mathrm{SD}=10.09)$ completed the survey. 76 had experience with VR. Ordinal data was analysed using non-parametric statistical tests, and was followed by pair-wise comparisons with Bonferroni corrected p-values.

\section{Comfort When Interrupting}

Figure 1 shows the comfort levels of interrupting friends and strangers across all settings. A Friedman test found significant differences between the comfort when interrupting a friend and stranger and between settings. Pairwise comparisons using Wilcoxon Signed Rank test with Bonferroni corrected p-values 
$(p<0.0018)$ revealed significant differences between the comfort interrupting a friend and a stranger across all settings. No significant differences were found between the comfort interrupting a friend across settings suggesting comfort when interrupting a friend is independent of setting. The only significant difference found between comfort when interrupting a stranger across the settings was Private and Public Transport. This difference may have occurred due to the interpretation of "private transport with a stranger" by our survey respondents. While car-sharing services like UberPool [30] do offer this scenario it may be difficult for respondents without experience using such services to imagine how they would react.

\section{Willingness to Adopt Interruption Strategies}

Figure 2 shows the combined scores for each interruption strategy across all settings. The results show a high willingness towards speaking to the VR user for both friends and strangers. Touch followed closely for friends but not for strangers. Peripheral devices performed similarly for both. The keyboard and gesture conditions performed poorly for both. 9 "Other" responses were submitted. 5 suggested novel interruptions such as "Stomping the ground to create vibrations" which highlights the playfulness bystanders can have when interrupting. 4 comments stated the bystander would linger or not interrupt the VR user.

A Friedman test showed significant differences between the combined interruption strategy scores. Pairwise comparisons using Wilcoxon Signed Rank test with Bonferroni corrected p-values $(p<0.0011)$ were conducted. The "Other" category was removed from the statistics as it was an optional entry. Significant differences between the willingness of bystanders to use the proposed strategy when interrupting friends and strangers was found in 18 of the 25 comparisons (Figure 3).

Pairwise comparisons of the strategies within friend across settings found significant differences between 8 of the 10 comparisons. No significant difference was found between "FriendTouch, Friend-Speak" and "Friend-Wave, Friend-Keyboard" highlighting touch as a comfortable strategy when interrupting friends. Pairwise comparisons of the strategies within stranger across settings significant differences between 8 of the 10 comparisons No significant difference was found between "Stranger-Touch, Stranger-Peripheral" and "Stranger-Wave, Stranger-Keyboard".

\section{LAB STUDY: INTERRUPTIONS IN PRACTICE}

A lab study further investigated how bystanders interrupt a known VR user in a private setting. Through post hoc interviews we aimed to better understand a bystander's rationale behind their interruption strategy. The setting was chosen to represent the most common location where VR is currently used - the home. Additionally, we sought to investigate the influence application choice had on the interruption strategy used. We hypothesized a more active application would deter bystanders from interrupting via touch but in practice found most used touch irrespective of application type.

\section{Design}

We used a between-subjects design with application type as the independent variable. 2 conditions were counterbalanced

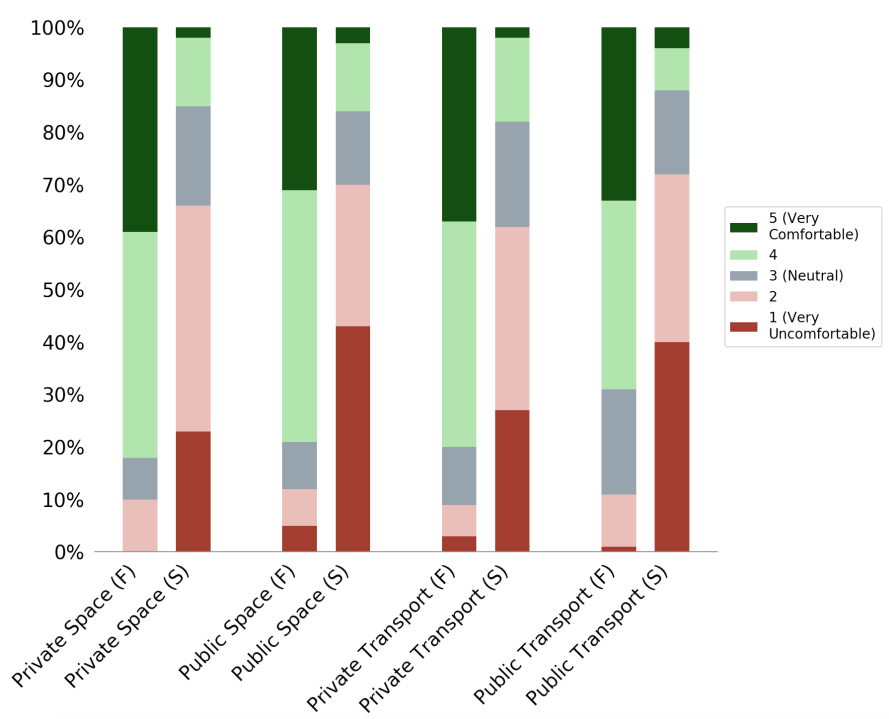

Figure 1. Comfort ratings when interrupting friends (F) or strangers (S) in public and private spaces, and in public and private transport. Results show significant differences in comfort when interrupting a friend and stranger but no difference when comparing across settings.

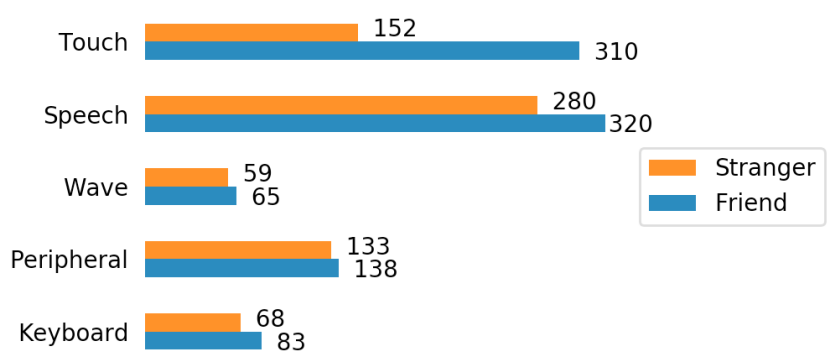

Figure 2. Combined scores from all settings for willingness to perform interruption strategies. Results show a high willingness to use speech when interrupting friends and strangers and touch when interrupting friends

\begin{tabular}{|l|c|c|c|c|c|}
\hline \multicolumn{1}{|c|}{$\boldsymbol{S}$} & Touch & Speak & Wave & Peripheral & Keyboard \\
\hline Touch & $<0.0011$ & 0.013664 & $<0.0011$ & $<0.0011$ & $<0.0011$ \\
\hline Speak & $<0.0011$ & $<0.0011$ & $<0.0011$ & $<0.0011$ & $<0.0011$ \\
\hline Wave & $<0.0011$ & $<0.0011$ & 0.330390 & $<0.0011$ & 0.035297 \\
\hline Peripheral & 0.317311 & $<0.0011$ & $<0.0011$ & 0.483840 & $<0.0011$ \\
\hline Keyboard & $<0.0011$ & $<0.0011$ & 0.773846 & $<0.0011$ & 0.025347 \\
\hline
\end{tabular}

Figure 3. P-values comparisons scores for interruption approaches for friends (F) and strangers ( $S$ ) shows no significant difference between friend-touch and stranger-speech

across participants: 1) a passive, seated experience (Epic Roller Coasters [1]), and 2) an active, standing experience (Beat Saber [10]). Epic Roller Coasters is an on-the-rails VR experience. Beat Saber is a music rhythm game where the player avoids in-game hazards by crouching or stepping to the left or right and uses slash-like motions to hit targets.

\section{Participants \& Procedure}

Participants were recruited using social media and mailing lists and were recruited in pairs to ensure they knew each other beforehand. 16 participants ( 5 females) in 8 pairs completed 
the study. Participants age ranged from 20 to $37(M=24.06$, $\mathrm{SD}=5.05$ ) with 10 reporting prior experience using VR headsets. Upon arrival the session's purpose was explained and participants were randomly assigned the role of VR user and bystander. The bystander, whose task was to interrupt the VR user, was removed from the room and given a consent form and demographics questionnaire. The VR user was fit with the headset and instructed to start the application. The experimenter then left the room. After 60 seconds the experimenter instructed the bystander to enter the room and interrupt the VR user. 60 seconds corresponded to approximately 1/3 through the Beat Saber song, where the VR user would be beginning a medium intensity slashing gesture section. Participants were not provided with any suggested interruption strategy and were left to devise their own approach. The interruption strategy used was observed and noted by the experimenter. The roles were then switched and the process repeated. After the participants had experienced both roles a semi-structured interview was conducted. Participants were interviewed together and were asked why they chose their particular interruption strategy and how they would act in different contexts (e.g. a more passive or active VR user).

\section{Apparatus}

An Oculus Quest headset was used during the study. Participants used the headset's onboard, open ear audio during the experiment. The headset volume was set to $53.3 \%$ (8 bars of 15) during the experiment which created some audio leakage but allowed the VR user to hear a verbal interruption.

\section{Limitations}

Interruption strategy is dependent on the urgency of the interruption. Our interruptions are fake by nature and cannot replicate the emotions behind a genuine urgent interruption. Participants were instructed to interrupt the VR user because they needed their attention. We attempted to create a neutral sense of urgency. Our study is limited to stationary applications and does not investigate the effect of room-scale applications. Participant comments suggest room-scale applications would cause a change in interruption strategy and future work could validate such claims. Finally as our VR users knew the interruption would occur and had a small time in VR comments made about their experience being interrupted are limited with follow-up work being necessary to document the VR user's perspective of being interrupted.

\section{LAB STUDY RESULTS}

Qualitative results are based on interview transcripts and observations by the experimenter. Interview transcripts were coded using initial coding [2] where participants' statements were assigned emergent codes over repeated cycles with the codes grouped using a thematic approach. Analysis of the observation notes followed the same approach. A single coder performed the coding. Three coding cycles were completed.

\section{Observed Interruption Strategies}

Our results found a combination of touch and speech (irrespective of order) was the preferred interruption strategy. Application type did not influence the choice of interruption strategy in our study. The strategies used were: Touch \& Speech (irrespective of order): 6 of 16; Speech only: 5 of 16; Playful Interruptions: 3 of 16; Touch only: 2 of 16.

Interestingly, 3 participants chose to improvise and create an unconventional interruption. 1 stealthily approached the VR user, hugged them and shouted their name. Another touched a standing VR user behind their knee while whispering in their ear using a fake voice. The third saw the bystander use a wooden stick to prod the VR user. We term these "playful interruptions" as the bystander's intent was not malicious but instead to act playfully. We distinguish between the use of speech and touch playfully in our count of used strategies as the bystander's intent differed from the conventional usage.

In total 11 participants approached the VR user when interrupting to either touch or speak from close proximity. 9 were confident in their approach while 3 took a slower, more cautious approach. 1 participant attempted a hesitant approach to touch the VR user before backing off and switching to a verbal interruption from afar. They said a "fear of being punched" by the VR caused their switch in strategy. Examining how bystanders used touch to interrupt, excluding the 3 playful interruptions, all lightly touched the VR user on the shoulder. 5 tapped the VR user on the shoulder while 3 lay their hand on the VR user's shoulder and did not remove it until the VR user took off the headset.

\section{Speech and Touch are Natural Interruption Strategies}

5 participants commented using speech or touch to interrupt was comfortable and a natural behaviour, P4: "I mean that's how you get someone's attention right, you say hello”. 4 said they used both to reassure the VR user. They did not want to make the VR user feel uncomfortable and thought verbally announcing their presence then using touch to indicate where they were located would be most comfortable for the VR user. P12: "I'd already got his attention but I just wanted to make sure that he knew where I was".

\section{Past Experience Influencing Interruption Strategy}

6 participants said prior experience (of lack of) with VR headsets influenced their choice of interruption strategy. 3 participants, unfamiliar with open ear audio, were uncertain if they would be heard if they spoke from afar due to the audio leakage given off by the headset. P4: "I thought the VR user wouldn't hear me, I came close because I could hear music”.

3 participants commented being interrupted first during the study influenced their interruption strategy. Specifically, they realised they could hear someone speaking to them over the headset's audio more clearly than they expected. This influenced them to use a verbal interruption which they then felt was sufficient for attracting attention. P14: "being the first one to use it I knew I could just talk and they would probably be able to hear me".

Prior experience being playfully interrupted was cited by 1 participant as their reason for using a playful interruption during the study. P5: "I was going towards what two of my friends were doing when I first tried VR". Another participant, who did not use or experience a playful interruption, commented 
they considered using one because of a past experience being interrupted playfully by a family member. P16: "I could have tapped their head, that works on me when my sisters do it".

\section{Relationship to the VR User Rather Than Setting}

11 participants said they would be less comfortable interrupting a stranger using VR and would change their approach, reinforcing the survey results. 1 participant who worked in a hospital speculated they might alter their approach depending on which of their colleagues they were interrupting. P10: "if it's one of the surgeons I'd be like 'excuse me' but if it was one of my other nurses I'd be tempted to tap them". Another discussed how they might begin with a verbal approach and escalate in intrusiveness should the less intrusive approach fail to get the person's attention. P6: "I'd probably put more emphasis on trying the audible first".

\section{Room-Scale VR May Influence Interruption Approach}

5 participants believed they would change their interruption strategy had a room-scale application, one where the user walks around the space, been used in the study. Participants felt there was little difference between the applications used in our study as our active application featured a mostly stationary VR user who could be observed, predicted and avoided unlike room-scale applications where the user would move unpredictably. P6: "if it was like a walk around sim where someone is walking around the space I'd feel less comfortable going up to them and tapping their shoulder".

\section{Personality Traits Influencing Interruption Strategy}

5 participant comments suggest the interrupter's personality may influence the choice of and willingness to perform an interruption strategy. 2 participants justified their use of playful interruptions as a desire to act playfully or mischievously. $P 2$ : "I was curious to see if I'd get punched or not". 1 participant, who experienced a playful interruption, commented why they would not use an unconventional approach. P6: "I don't have the confidence to inflict that kind of stress on someone". Interestingly, 2 participants expressed an unwillingness to interrupt the VR user if their reason for interruption was non-urgent, commenting they might linger or leave and return later when the person might no longer be in VR. P14: "just go away and let them do their thing, I'll come back later, you [VR user] are busy obviously".

\section{SURVEY: RESPONSE TO OPEN EAR AUDIO}

With our results indicating a high willingness to use verbal interruptions and Ghosh et al's identification of 8 scenarios where VR users desire more auditory awareness [7] open ear audio looks promising. However, as the response to open ear audio in VR headsets is unclear we investigated the response to it from owners of open ear audio equipped VR headsets.

\section{Survey Design}

We developed a survey to investigate the usage and response to open ear audio from owners of open ear audio equipped VR headsets. The survey first asked respondents if they used the open ear audio functionality of their respective headsets $(\mathrm{Yes} / \mathrm{No}$ ). Respondents who replied No were asked what they used for audio, why they did not use open ear audio and if they could imagine situations where they would use it. Respondents who replied Yes were asked to indicate how often they used open ear audio using a 5-point scale (1=very rarely; 5=very often), in which situations they typically did and did not use it and to rate their experience with it on a 5 -point scale (1=very poor; 5=very good). An optional, open text allowed respondents to give additional comments about open ear audio.

\section{SURVEY RESULTS}

We distributed the survey through mailing lists and social media. 76 (15 female) respondents aged between 16 and 65 $(\mathrm{M}=31.07, \mathrm{SD}=11.92)$ completed the survey. Respondents were asked to indicate their prior experience with VR headsets using a 5-point scale (1=very little; 5=a lot), $(\mathrm{M}=3.63$, $\mathrm{SD}=1.23)$. Respondent qualitative answers were coded using initial coding [2] where respondents' statements were assigned emergent codes over repeated cycles with the codes grouped using a thematic approach. A single coder performed the coding. Three coding cycles were completed. Our survey found $26(34.2 \%)$ of respondents did not use the open ear audio of their respective headsets while $50(65.8 \%)$ did.

\section{Respondents Who Do Not Use Open Ear Audio}

All 26 respondents who did not use open ear audio used some type of headphones instead ( 8 in-ear, 12 over-the-ear, 8 on-theear, 1 bone conduction). Reasons for not using open ear audio were mixed. 16 said poor audio quality due to factors like low maximum volume or a lack of bass, P12: "Lack of overall fidelity. No bass. Low max output volume.”. 12 felt open ear audio inhibited sense of immersion in VR due to the reduced isolation from their surroundings, P9: "Not immersive enough, I don't want to be able to hear my surroundings". 5 said audio leakage being disruptive to nearby people, P3: "I play late into the night and don't want to disturb the house" while 2 said audio leakage was a privacy concern, P5: "I use voice comms and don't really want my family listening in”. Few could imagine a scenario where they would use open ear audio though 5 identified social situations as a use case, $P 7$ : "if I'm hanging with family or friends and we're sharing the headset".

\section{Respondents Who Use Open Ear Audio}

Of the 50 respondents who said they used open ear audio $80 \%$ said they used it often or very often: Very Rarely $=0$ of 50, Rarely $=3$ of 50, Sometimes $=7$ of 50, Often $=8$ of 50, Very Often $=32$ of 50 (Appendix 2). 60\% reported a good or very good experience using it: Very Poor $=0$ of 50, Poor $=8$ of 50, Average $=12$ of 50, Good $=13$ of 50, Very Good $=17$ of 50 (Appendix 3). When asked which situations they typically used it 19 respondents replied "all" with a further 10 respondents claiming the majority of the time. 7 identified social situations as their primary use of it, P60: "When there's a group of friends around so the person wearing the headset can hear us and we can hear the audio". When asked when they did not use open ear audio 15 respondents said when they wanted to maximise immersion within an experience, P42: "When I want to immerse myself into the game". 10 said they would not use it if they thought it would be disruptive 
to someone nearby and 2 when they were concerned about their privacy due to audio leakage from the headset. In the additional comments 7 respondents critiqued the bass in the audio, P45: "Needs beefier bass". 6 said an accessory should be included to provide an attachable over-the-ear audio system, P69: "I'd like clip-on over-ear speakers as an accessory".

\section{DISCUSSION}

\section{The Interruption of VR Users}

Social interruption methods (speech / touch) were the preferred methods of interruptions in our survey and lab study. The majority of bystanders were not deterred from using either to interrupt despite prior work speculating they would be [33]. Interestingly, during the lab study several participants said audio leakage, caused by the open ear audio design of the headset, made them unsure if a verbal interruption would be heard by the VR user. This suggests a potential false affordance due to the audio leakage created by the open ear audio design. Open ear audio aims to provide the VR user with an increased awareness of verbal interruptions but the bystander is deterred from verbally interrupting because they assume they will not be heard because of the audio leakage.

Furthermore, participant comments made during our lab study suggest some perceive a VR user as someone who had "gone through the trouble of putting on a headset" and had a specific purpose they wished to use VR for so would not want to be disturbed. If their reason for interruption was non-urgent these participants felt they might leave and return later rather than interrupt the VR user. A similar result was seen by George et al. [4] who documented participants who disobeyed instructions to interrupt a VR user and chose to wait until they had finished using the headset before interrupting. What motivates this behaviour and perception and comparisons between the perception of interrupting a VR user and smartphone or PC user is a potential direction for future work.

\section{Playful Interruptions of VR Users}

During the lab study 3 participants chose to take advantage of the VR user's vulnerable state and improvise a novel interruption strategy. Such interruptions are a promising emergence from our study and future work could explicitly elicit or integrate them into follow-up work. Playful interruptions, unlike system notifications, can be personal in ways general purpose notifications cannot. For example, a playful interruption might emerge naturally between family members. For some this unconventional, esoteric interruption method that develops over time becomes an extension of their relationship and human desire to act playfully with one another [20,21].

However, playful interruptions are abusive of the VR user's sensitive state. While no VR user in our study reacted negatively to being playfully interrupted not all VR users will react as such. Notification systems should be available for users who wish to reduce the risk of being abused. These should provide users with systems, such as those proposed by McGill et al. [13] or Simone [25], to automatically be informed of copresence. Such notifications are not present in consumer VR headsets and further work is necessary in this area.

\section{The On-going Need for Accessible Interruptions}

Work has begun to identify accessibility issues in VR [16] and investigated methods to assist users who would otherwise be inhibited in their enjoyment of or access to VR $[35,36,15]$. Primarily this work has considered how VR can be made more accessible but future work should consider how the technology's use might create issues. While interrupting a VR user using speech or touch may be sufficient for the majority of bystanders some will be unable to use either. In the related work section we highlighted one use case where an individual modified the HTC Vive Knock notification to be triggered using a bluetooth remote [28]. As the individual's partner had mobility issues they were unable to use the standard method of triggering the notification. This custom solution provided an easy way for them to trigger the notification. This is a very real use case existing VR headsets do not cater for. Future work should consider how VR can be made more accessible but should also consider how the technology's use by members of the household, workplace, etc. can create issues for some and how these may be solved.

\section{Open Ear Audio in VR Headsets}

Prior work by Ghosh et al. [7] highlighted the desire of VR users for increased awareness of nearby sounds. In our view, open ear audio fulfils this need though implementation specific issues such as "lack of bass" and "too quiet" require improvement. Our survey found $65.8 \%$ of respondents used the open ear audio of their respective headsets with $52.6 \%$ of respondents using it regularly. $60 \%$ of those who used open ear audio rated it positively but this only $39.5 \%$ of the total survey respondents. There is much room for improvement in the implementation of open ear audio and future work should investigate how to improve and iterate on the technology. Iterations might involve dynamically adjust the volume in response to sounds in the user's nearby area or amplify sounds from the user's surrounding environment. However, open ear audio will not satisfy all users. Some will always opt for the sound isolation and audio experience provided by headphones. While others who feel uncomfortable wearing headphones or desire more awareness of their surroundings will use the technology.

\section{CONCLUSION}

This paper documents a survey and lab study which investigated the interruption of a VR user from the interrupter's perspective. Our survey found an interrupter's relationship to the VR user is more influential on comfort and choice of interruption strategy than setting. Our lab study found a combination of speech and touch was the preferred interruption approach and documented the use of improvised unconventional, novel interruptions we term "playful interruptions". Due to the willingness to use and high usage of verbal interruptions in our results we conducted on a survey into the consumer response to open ear audio in VR headsets. We found open ear audio was used regularly by $52.6 \%$ of our respondents $60 \%$ of who rate their experience with it positively.

\section{REFERENCES}

[1] B4T Games. 2018. Epic Roller Coasters. Game [Oculus Quest / PC]. (7 March 2018). 
[2] Strauss A. L. Corbin J. M. 1998. Basics of qualitative research: techniques and procedures for developing grounded theory. SAGE Publications, Inc.

[3] Ceenu George, Manuel Demmler, and Heinrich Hussmann. 2018. Intelligent Interruptions for IVR: Investigating the Interplay Between Presence, Workload and Attention. In Extended Abstracts of the $2018 \mathrm{CHI}$ Conference on Human Factors in Computing Systems (CHI EA '18). ACM, New York, NY, USA, Article LBW511, 6 pages. DOI : http://dx.doi.org/10.1145/3170427.3188686

[4] Ceenu George, Philipp Janssen, David Heuss, and Florian Alt. 2019. Should I Interrupt or Not?: Understanding Interruptions in Head-Mounted Display Settings. In Proceedings of the 2019 on Designing Interactive Systems Conference (DIS '19). ACM, New York, NY, USA, 497-510. DOI :

http://dx.doi.org/10.1145/3322276.3322363

[5] Ceenu George, Mohamed Khamis, Emanuel von Zezschwitz, Marinus Burger, Henri Schmidt, Florian Alt, and Heinrich Hussmann. 2017. Seamless and Secure VR: Adapting and Evaluating Established Authentication Systems for Virtual Reality. In Proceedings of the Network and Distributed System Security Symposium (NDSS 2017) (USEC '17). NDSS. DOI : http://dx.doi.org/10.14722/usec.2017.23028

[6] Jeff Gerstmann. 2019. Giant Bombcast 584: Bend It Like Blevins (Oculus Quest Review). https://youtu.be/80jQut7cp-k?t=2268. (21 May 2019). Accessed: 2020-02-13.

[7] S. Ghosh, L. Winston, N. Panchal, P. Kimura-Thollander, J. Hotnog, D. Cheong, G. Reyes, and G. D. Abowd. 2018. NotifiVR: Exploring Interruptions and Notifications in Virtual Reality. IEEE Transactions on Visualization and Computer Graphics 24, 4 (April 2018), 1447-1456. DOI:

http://dx.doi.org/10.1109/TVCG. 2018.2793698

[8] Jan Gugenheimer, Christian Mai, Mark McGill, Julie Williamson, Frank Steinicke, and Ken Perlin. 2019. Challenges Using Head-Mounted Displays in Shared and Social Spaces. In Extended Abstracts of the 2019 CHI Conference on Human Factors in Computing Systems (CHI EA '19). ACM, New York, NY, USA, Article W19, 8 pages. DOI :

http://dx.doi.org/10.1145/3290607.3299028

[9] Philipp Hock, Sebastian Benedikter, Jan Gugenheimer, and Enrico Rukzio. 2017. CarVR: Enabling In-Car Virtual Reality Entertainment. In Proceedings of the 2017 CHI Conference on Human Factors in Computing Systems (CHI '17). ACM, New York, NY, USA, 4034-4044. DOI:

http://dx.doi.org/10.1145/3025453.3025665

[10] Hyperbolic Magnetism. 2018. Beat Saber. Game [Oculus Quest / PSVR / PC]. (1 May 2018).
[11] Katrina_18. 2019. The Rift S is way way WAY better than you think. https:

//www.reddit.com/r/virtualreality/comments/b7c22c/ the_rift_s_is_way_way_way_better_than_you_think. (2 April 2019). Accessed: 2020-02-13.

[12] Christian Mai, Tim Wiltzius, Florian Alt, and Heinrich Hußmann. 2018. Feeling Alone in Public: Investigating the Influence of Spatial Layout on Users' VR Experience. In Proceedings of the 10th Nordic Conference on Human-Computer Interaction (NordiCHI '18). ACM, New York, NY, USA, 286-298. DOI : http://dx.doi.org/10.1145/3240167.3240200

[13] Mark McGill, Daniel Boland, Roderick Murray-Smith, and Stephen Brewster. 2015. A Dose of Reality: Overcoming Usability Challenges in VR Head-Mounted Displays. In Proceedings of the 33rd Annual ACM Conference on Human Factors in Computing Systems (CHI '15). ACM, New York, NY, USA, 2143-2152. DOI : http://dx.doi.org/10.1145/2702123.2702382

[14] Mark McGill, Alexander Ng, and Stephen Brewster. 2017. I Am The Passenger: How Visual Motion Cues Can Influence Sickness For In-Car VR. In Proceedings of the 2017 CHI Conference on Human Factors in Computing Systems (CHI '17). ACM, New York, NY, USA, 5655-5668. DOI:

http://dx.doi.org/10.1145/3025453.3026046

[15] M. Mirzaei, P. Kán, and H. Kaufmann. 2020. EarVR: Using Ear Haptics in Virtual Reality for Deaf and Hard-of-Hearing People. IEEE Transactions on Visualization and Computer Graphics 26, 5 (2020), 2084-2093.

[16] M. Mott, E. Cutrell, M. Gonzalez Franco, C. Holz, E. Ofek, R. Stoakley, and M. Ringel Morris. 2019. Accessible by Design: An Opportunity for Virtual Reality. In 2019 IEEE International Symposium on Mixed and Augmented Reality Adjunct (ISMAR-Adjunct). 451-454.

[17] Oculus and Facebook. 2018. Oculus Go. Hardware device. (May 2018).

[18] Oculus and Facebook. 2019a. Oculus Quest. Hardware device. (May 2019).

[19] Oculus and Facebook. 2019b. Oculus Rift S. Hardware device. (May 2019).

[20] Jaak Panksepp. 1998. Affective neuroscience: The foundations of human and animal emotions. Oxford University Press, New York, NY, US. xii, 466-xii, 466 pages.

[21] Jaak Panksepp, Steve Siviy, and Larry Normansell. 1984. The psychobiology of play: Theoretical and methodological perspectives. Neuroscience \& Biobehavioral Reviews 8, 4 (1984), 465 - 492. DOI : http://dx.doi.org/https: //doi.org/10.1016/0149-7634(84)90005-8 
[22] Rufat Rzayev, Sven Mayer, Christian Krauter, and Niels Henze. 2019. Notification in VR: The Effect of Notification Placement, Task and Environment. In Proceedings of the Annual Symposium on Computer-Human Interaction in Play (CHI PLAY '19). Association for Computing Machinery, New York, NY, USA, 199-211. DOI:

http://dx.doi.org/10.1145/3311350.3347190

[23] Maria Sanchez-Vives and Mel Slater. 2005. From presence to consciousness through virtual reality. Nature reviews. Neuroscience 6 (05 2005), 332-9. DOI:

http://dx.doi.org/10.1038/nrn1651

[24] Valentin Schwind, Jens Reinhardt, Rufat Rzayev, Niels Henze, and Katrin Wolf. 2018. Virtual Reality on the Go?: A Study on Social Acceptance of VR Glasses. In Proceedings of the 20th International Conference on Human-Computer Interaction with Mobile Devices and Services Adjunct (MobileHCI '18). ACM, New York, NY, USA, 111-118. DOI :

http://dx.doi.org/10.1145/3236112.3236127

[25] A. L. Simeone. 2016. The VR motion tracker: visualising movement of non-participants in desktop virtual reality experiences. In 2016 IEEE 2nd Workshop on Everyday Virtual Reality (WEVR). 1-4. DOI : http://dx.doi.org/10.1109/WEVR.2016.7859535

[26] Daniel J Simons and Christopher F Chabris. 1999. Gorillas in Our Midst: Sustained Inattentional Blindness for Dynamic Events. Perception 28, 9 (1999), 1059-1074. DOI : http://dx.doi.org/10.1068/p281059 PMID: 10694957.

[27] Takahiro Tanaka and Kinya Fujita. 2011. Study of User Interruptibility Estimation Based on Focused Application Switching. In Proceedings of the ACM 2011 Conference on Computer Supported Cooperative Work (CSCW'11). ACM, New York, NY, USA, 721-724. DOI : http://dx.doi .org/10.1145/1958824.1958954

[28] thyazide. 2019. Remote Knock Knock. https://www .reddit. com/r/Vive/comments/8r8k85/remote_ knock_knock/. (3 April 2019). Accessed: 2020-02-13.

[29] tirehabitat. 2019. WORST AUDIO DECISION EVER Oculus Rift S.

https://www . reddit. com/r/oculus/comments/brdw38/ worst_audio_decision_ever_oculus_rift_s. (1 June 2019). Accessed: 2020-02-13.

[30] Uber. 2016. UberPool. Website. (6 August 2016). https://www.uber.com/en-GB/ride/uberpool/.

[31] Valve. 2016. HTC Vive Knock Notification. Software. (26 May 2016). https://uploadvr.com/new-knock-knockfeature-lets-you-know-someone-needs-attention/.

[32] Valve. 2019. Valve Index. Hardware device. (June 2019).

[33] Julie R. Williamson, Mark McGill, and Khari Outram. 2019. PlaneVR: Social Acceptability of Virtual Reality for Aeroplane Passengers. In Proceedings of the 2019 CHI Conference on Human Factors in Computing
Systems (CHI '19). ACM, New York, NY, USA, Article 80,14 pages. DOI :

http://dx.doi.org/10.1145/3290605.3300310

[34] André Zenner, Marco Speicher, Sören Klingner, Donald Degraen, Florian Daiber, and Antonio Krüger. 2018. Immersive Notification Framework: Adaptive \&\#38; Plausible Notifications in Virtual Reality. In Extended Abstracts of the 2018 CHI Conference on Human Factors in Computing Systems (CHI EA '18). ACM, New York, NY, USA, Article LBW609, 6 pages. DOI : http://dx.doi.org/10.1145/3170427.3188505

[35] Yuhang Zhao, Cynthia L. Bennett, Hrvoje Benko, Edward Cutrell, Christian Holz, Meredith Ringel Morris, and Mike Sinclair. 2018. Enabling People with Visual Impairments to Navigate Virtual Reality with a Haptic and Auditory Cane Simulation. In Proceedings of the 2018 CHI Conference on Human Factors in Computing Systems (CHI '18). Association for Computing Machinery, New York, NY, USA, Article Paper 116, 14 pages. DOI : http://dx.doi.org/10.1145/3173574. 3173690

[36] Yuhang Zhao, Edward Cutrell, Christian Holz, Meredith Ringel Morris, Eyal Ofek, and Andrew D. Wilson. 2019. SeeingVR: A Set of Tools to Make Virtual Reality More Accessible to People with Low Vision. In Proceedings of the 2019 CHI Conference on Human Factors in Computing Systems (CHI'19). Association for Computing Machinery, New York, NY, USA, Article Paper 111, 14 pages. DOI :

http://dx.doi.org/10.1145/3290605.3300341 
APPENDIX

Appendix 1
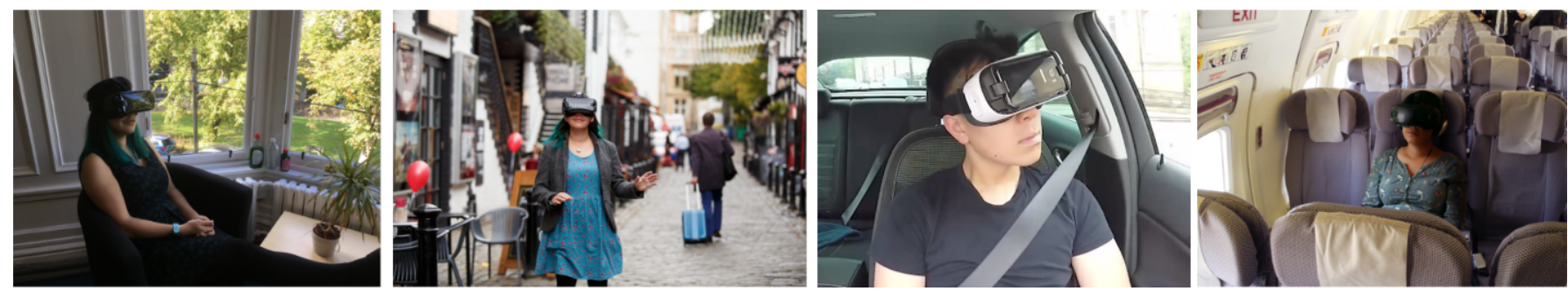

Figure 4. Images used in the survey to represent a VR user in the four chosen settings. Settings from left to right are: private space, public space, private transport, public transport

\section{Appendix 2}

$$
\begin{aligned}
& \text { Very Rarely } 0 \\
& \text { Rarely } 3 \\
& \text { Sometimes } 7 \\
& \text { Often } \quad 8 \\
& \text { Very Often }
\end{aligned}
$$

Figure 5. The frequency the 50 respondents who identified as open ear audio users use the technology

\section{Appendix 3}

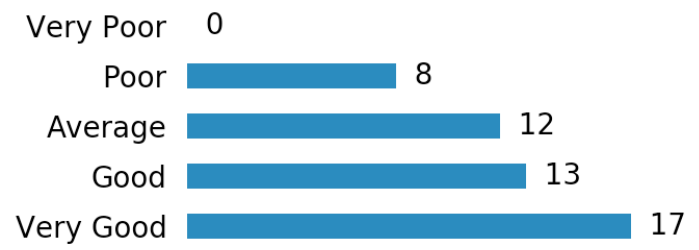

Figure 6. The ratings the 50 the 50 respondents who identified as open ear audio users rate their experience with the technology 